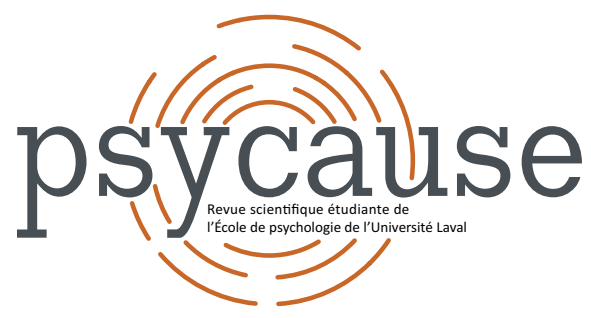

\section{PSYCAUSE}

Revue scientifique étudiante de l'École de psychologie de l'Université Laval

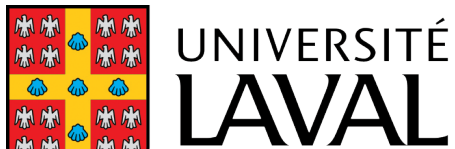

Faculté des sciences sociales École de psychologie

\title{
EFFETS DES PROCÉDURES DE REVASCULARISATION SUR L'ANXIÉTÉ, L'ANXIÉTÉ CARDIAQUE ET LA DÉPRESSION
}

Claudia-Béatrice RATTÉ1-2*, Justine PAGÉ1-2, Jeanne PRÉMONT-BOULET ${ }^{1-2}$,

Marie-France DE LAFONTAINE 1 -2-23, Clermont DIONNE ${ }^{4}$, Paul POIRIER ${ }^{3}$, Isabelle DENIS ${ }^{1-2-5}$ et Guillaume FOLDES-BUSQUE ${ }^{1-2-3}$

1 École de psychologie, Université Laval

${ }^{2}$ Centre de recherche du CISSS de Chaudière-Appalaches

${ }^{3}$ Centre de recherche de l'Institut universitaire de cardiologie et de pneumologie de Québec (IUCPQ),

${ }^{4}$ Centre de recherche du CHU de Québec

${ }^{5}$ Centre de recherche universitaire sur les jeunes et les familles (CRUJeF)

*claudia-beatrice.ratte.1@ulaval.ca

\section{Pour citer l'article}

Ratté, C.-B., Pagé, J., Prémont-Boulet, J., de Lafontaine, M.-F., Dionne, C., Poirier, P., Denis, I., \& Foldes-Busque, G. (2021). Effets des procédures de revascularisation sur l'anxiété, I'anxiété cardiaque et la dépression. Psycause: Revue scientifique étudiante de l'École de psychologie de I'Université Laval, 11(2), 9-11. 
Olff, M., Langeland, W., Draijer, N., \& Gersons, B. P. R. (2007). Gender differences in posttraumatic stress disorder. Psychological Bulletin, 133(2), 183-204. https://doi. org/10.1037/0033-2909.133.2.183

Van Ameringen, M., Mancini, C., Patterson, B., \& Boyle, M. H. (2008). Post-traumatic stress disorder in Canada. CNS Neuroscience \& Therapeutics, 14(3), 171-181. https://doi. org/10.1111/j.1755-5949.2008.00049.x

Weathers, F. W., Blake, D. D., Schnurr, P. P., Kaloupek, D. G., Marx, B. P., \& Keane, T. M. (2013). The PTSD Checklist for DSM-5 (PCL-5). PTSD: National Center for PTSD. https:// www.ptsd.va.gov/professional/assessment/adult-sr/ptsdchecklist.asp

\title{
Pour citer l'article
}

Bédard, S., Gagner, E., Proulx-Villeneuve, J., Binet, É., \& Belleville, G. (2021). Différences de genre dans la présentation des symptômes post-traumatiques chez les évacués de feux de forêt. Psycause: Revue scientifique étudiante de l'École de psychologie de I'Université Laval, 11(2), 7-9.

\section{EFFETS DES PROCÉDURES DE REVASCULARISATION SUR L'ANXIÉTÉ, L'ANXIÉTÉ CARDIAQUE ET LA DÉPRESSION}

\author{
Claudia-Béatrice RATTÉ1-2*, Justine PAGÉ1-2, Jeanne PRÉMONT-BOULET ${ }^{1-2}$, Marie-France DE LAFONTAINE ${ }^{1-2-3}$, \\ Clermont DIONNE ${ }^{4}$, Paul POIRIER ${ }^{3}$, Isabelle DENIS ${ }^{1-2-5}$ et Guillaume FOLDES-BUSOUE ${ }^{1-2-3}$ \\ ${ }^{1}$ École de psychologie, Université Laval, ${ }^{2}$ Centre de recherche du CISSS de Chaudière-Appalaches, ${ }^{3}$ Centre de recherche \\ de I'Institut universitaire de cardiologie et de pneumologie de Québec (IUCPQ), ${ }^{4}$ Centre de recherche du CHU de Québec, \\ ${ }^{5}$ Centre de recherche universitaire sur les jeunes et les familles (CRUJeF) \\ *claudia-beatrice.ratte.1@ulaval.ca
}

Mots-clés : pontage aortocoronarien, intervention coronarienne percutanée, anxiété, dépression, anxiété cardiaque

L'intervention coronarienne percutanée (ICP) et le pontage aortocoronarien (PAC) sont deux procédures de revascularisation pouvant atténuer les symptômes de la maladie coronarienne athérosclérotique (MCAS), une maladie chronique caractérisée par l'occlusion d'une ou plusieurs artères coronaires (Lusis, 2012). L'ICP consiste à insérer un cathéter muni d'un ballon à son extrémité et à le gonfler à l'endroit obstrué de l'artère coronaire afin d'en rétablir le diamètre (Chhabra et al., 2020), alors que le PAC est une chirurgie majeure plus invasive qui consiste à greffer un vaisseau sanguin au niveau de l'artère obstruée afin de créer une nouvelle voie de circulation sanguine (Centre hospitalier universitaire vaudois, 2017). La procédure choisie dépend notamment de la sévérité, de la localisation de l'occlusion ainsi que de l'état de santé du patient (Neumann et al., 2019).

Le type de revascularisation influencerait les symptômes d'anxiété et de dépression post-intervention, mais aucun consensus n'est établi quant à leur trajectoire (p. ex. Boudrez et De Backer, 2001 ; Gu et al., 2016). De plus, à la connaissance des auteurs, aucune étude ne rapporte comment l'anxiété cardiaque, la peur des sensations cardiovasculaires (p. ex., palpitations cardiaques) causée par l'anticipation de conséquences indésirables (p. ex., arrêt cardiaque; Chiasson et al., 2019), serait affectée par la revascularisation. L'objectif de cette étude est de comparer les trajectoires d'anxiété, d'anxiété cardiaque et de dépression entre I'ICP et le
PAC au moment post-intervention et trois mois plus tard. L'hypothèse postulée est qu'une diminution de l'anxiété, de l'anxiété cardiaque et de la dépression sera observée trois mois post-intervention pour les deux procédures et qu'elle sera plus accentuée pour le PAC.

\section{Méthode}

L'échantillon se compose de 200 participants (65 \pm 9 ans; 154 hommes) revascularisés pour une MCAS (ICP $=99$; $P A C=101)$ et recrutés consécutivement à I'IUCPQ. Cet échantillon est représentatif de la population atteinte d'une MCAS, qui comporte une majorité d'hommes (Lusis, 2012). Les participants présentant un problème de communication sévère ou toute autre condition médicale ( $p$. ex., trouble psychotique) pouvant nuire à la validité de l'étude n'étaient pas admissibles. L'Échelle hospitalière d'anxiété et de dépression (ÉHAD; Roberge et al., 2013) et le Questionnaire d'anxiété cardiaque (QAC; Chiasson et al., 2019) ont été auto-administrés afin de mesurer les symptômes d'anxiété, de dépression et d'anxiété cardiaque dans les 30 jours suivant la revascularisation et trois mois plus tard. L'ÉHAD comporte deux sous-échelles évaluant l'anxiété (ÉHAD-Anxiété) et la dépression (ÉHAD-Dépression). Le QAC évalue l'attention portée aux sensations thoraciques, l'évitement des activités cardiovasculaires, la peur des symptômes et la recherche de réassurance. 


\section{Résultats et discussion}

Les participants ayant subi une ICP présentent des scores significativement plus élevés à l'ÉHAD-Anxiété que ceux ayant subi un PAC aux deux temps de mesure (voir Tableau 1). Ce résultat corrobore les écrits suggérant que l'ICP serait perçue comme une réparation de l'artère malade en raison de son caractère moins invasif, tandis que le PAC serait perçu comme un remplacement puisqu'il s'agit d'une opération majeure (Akgul, 2019), ce qui pourrait affecter le niveau d'anxiété postopératoire. Ainsi, le patient percevrait I'ICP comme moins efficace que le PAC et, par conséquent, vivrait davantage de détresse psychologique liée au pronostic de la MCAS (Fiabane et al., 2014).

Les scores au QAC étaient significativement moins élevés lors du suivi à trois mois qu'en post-intervention pour les deux procédures. Cette diminution s'expliquerait par l'effet bénéfique de la revascularisation sur les symptômes cardiovasculaires ( $p$. ex. douleur thoracique), ce qui réduirait l'anxiété liée à ceux-ci.

\section{Tableau 1}

Comparaison des scores à l'ÉHAD-Anxiété, à l'ÉHAD-Dépression et au QAC en post-intervention et trois mois à la suite de l'intervention de revascularisation.

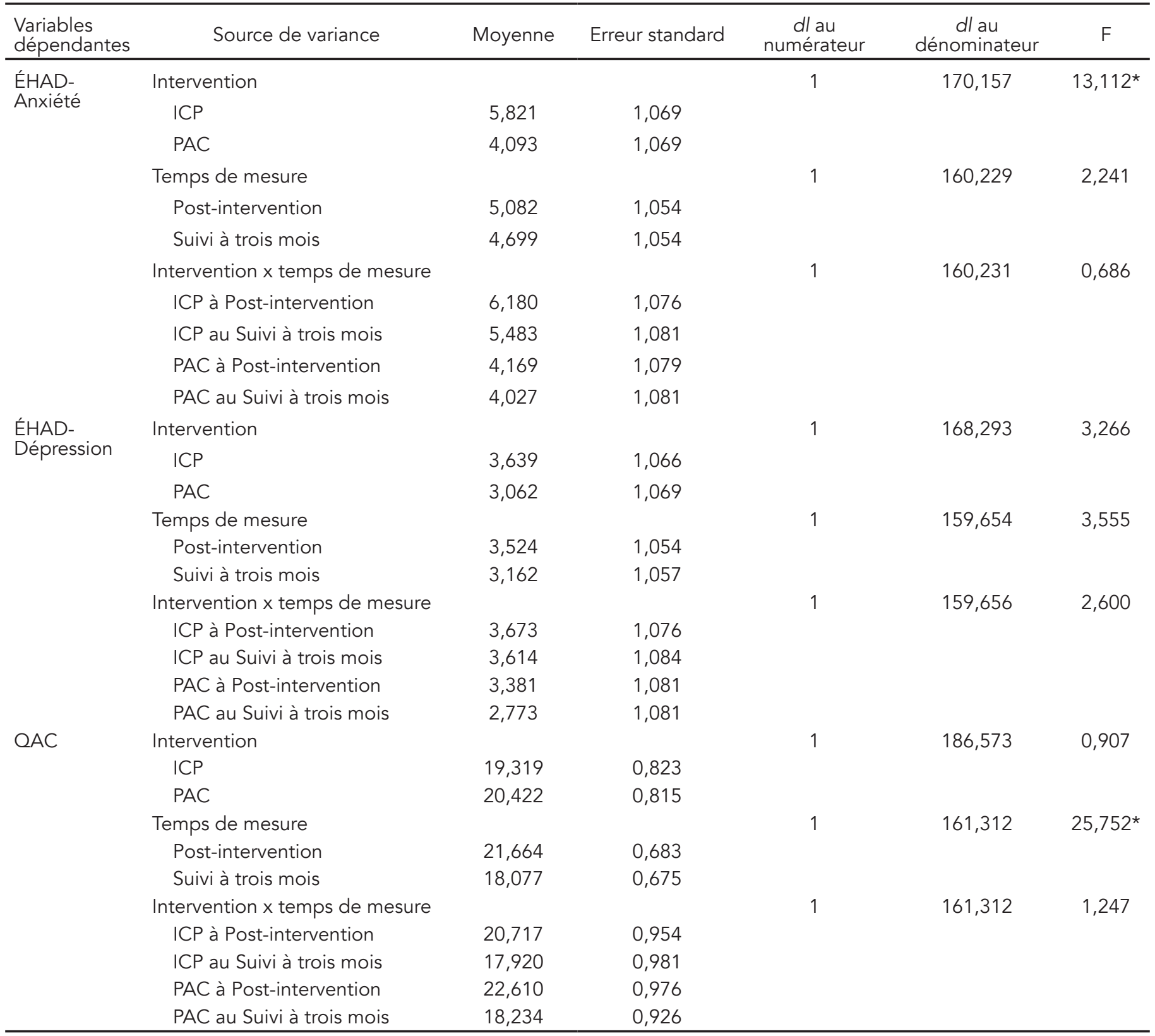

Notes: ÉHAD-Anxiété = Échelle hospitalière d'anxiété et de dépression, sous-échelle d'anxiété; QAC = Questionnaire d'anxiété cardiaque; ÉHADDépression = Échelle hospitalière d'anxiété et de dépression, sous-échelle de dépression; ICP = Intervention coronarienne percutanée; PAC $=$ Pontage aortocoronarien. $* p<.05$ 
Les différences au niveau de l'anxiété entre I'ICP et le PAC suggèrent que des suivis cliniques adaptés à l'intervention subie par le patient seraient nécessaires et que la perception de celui-ci devrait être considérée afin de gérer la détresse psychologique liée à la revascularisation. II serait également pertinent d'évaluer les bénéfices d'un programme de psychoéducation visant à moduler les croyances erronées des patients quant aux procédures de revascularisation.

\section{Références}

Akgul, E. (2019). Anxiety and depression are common in heart patients. Well is there any interaction between the type of intervention and anxiety/depression level? Heart patients need psychiatric rehabilitation. Journal of Evidence-Based Psychotherapies, 19(1), 141-151. https://doi.org/10.24193/ jebp.2019.1.9

Boudrez, H., \& De Backer, G. (2001). Psychological status and the role of coping style after coronary artery bypass graft surgery. Results of a prospective study. Quality of life research: an international journal of quality of life aspects of treatment, care and rehabilitation, 10(1), 37-47. https:// doi.org/10.1023/a 1016697719078

Centre hospitalier universitaire vaudois. (2017). Pontage avec circulation extra-corporelle. https://www.chuv.ch/ fr/chirurgie-cardiaque/ccv-home/patients-et-familles/ nos-techniques/chirurgie-coronaire/pontage-avec-circulation-extra-corporelle

Chhabra, L., Zain, M. A., \& Siddiqui, W. J. (2020). Angioplasty. Dans StatPearls. StatPearls Publishing.
Chiasson, C., Bisson-Bernatchez, E., Turcotte, S., Tremblay, M.-A., Denis, I., \& Foldes-Busque, G. (2019). Validation of the French-Canadian version of the Cardiac Anxiety Questionnaire (CAQ-FR). Canadian Journal of Behavioural Science / Revue canadienne des sciences du comportement, 51(2), 100-104. https://doi.org/10.1037/cbs0000123

Fiabane, E., Giorgi, I., Candura, S. M., \& Argentero, P. (2014). Psychological and work stress assessment of patients following angioplasty or heart surgery: Results of 1-year follow-up study. Stress and Health: Journal of the International Society for the Investigation of Stress, 31(5), 393-402. http://doi.org/10.1002/smi.2564

Gu, G., Zhou, Y., Zhang, Y., \& Cui, W. (2016). Increased prevalence of anxiety and depression symptoms in patients with coronary artery disease before and after percutaneous coronary intervention treatment. BMC Psychiatry, 16(1), 259. https://doi.org/10.1186/s12888-016-0972-9

Lusis, A. J. (2012). Genetics of atherosclerosis. Trends in genetics: TIG, 28(6), 267-275. https://doi.org/10.1016/j. tig.2012.03.001

Neumann, F. J., Sousa-Uva, M., Ahlsson, A., Alfonso, F., Banning, A. P., Benedetto, U., Byrne, R. A., Collet, J. P., Falk, V., Head, S. J., Jüni, P., Kastrati, A., Koller, A., Kristensen, S. D., Niebauer, J., Richter, D. J., Seferovic, P. M., Sibbing, D., Stefanini, G. G., Windecker, S., ... ESC Scientific Document Group (2019). 2018 ESC/EACTS Guidelines on myocardial revascularization. European heart journal, 40(2), 87-165. https://doi.org/10.1093/eurheartj/ehy394

Roberge, P., Doré, I., Menear, M., Chartrand, É., Ciampi, A., Duhoux, A., \& Fournier, L. (2013). A psychometric evaluation of the French Canadian version of the Hospital Anxiety and Depression Scale in a large primary care population. Journal of Affective Disorders, 147(1-3), 171-179. https://doi.org/10.1016/j.jad.2012.10.029

\section{Pour citer l'article}

Ratté, C.-B., Pagé, J., Prémont-Boulet, J., de Lafontaine, M.-F., Dionne, C., Poirier, P., Denis, I., \& Foldes-Busque, G. (2021). Effets des procédures de revascularisation sur l'anxiété, I'anxiété cardiaque et la dépression. Psycause: Revue scientifique étudiante de l'École de psychologie de l'Université Laval, 11(2), 9-11. 